\title{
Design and Development of Multifunction Map Software Based on Android Platform
}

\author{
Lu Qingru ${ }^{\mathrm{a}}$, Xin Haiyan ${ }^{\mathrm{b}}$,Huang $\mathrm{Hui}^{\mathrm{c}}$, Geng Yunlong \\ Department of Electronics, Chengxian Institute,Southeast University,Nanjing Jiangsu, China, \\ 210000
}

silver_1120@163.com,983511889@qq.com, huanghui_1225@163.com

\begin{abstract}
Keywords: Mobile Internet, Android development, Weather Forecast, Free API
Abstract. With the general use of smart phones, mobile phone software development becomes more and more popular. This paper introduces the multifunction map software developed under the Android system based on the free API provided by A Map Software. The paper explains the development environment and the main process of the software design, which realizes the functions of automatic positioning, hot point searching, bus route querying, travel route searching, advertising and weather forecasting under three map models.
\end{abstract}

\section{Introduction}

Mobile communication and the internet have become the two businesses which are the world's fastest developing, have the biggest market potential, and the most attractive prospects. Smart phones as mobile Internet portal, the operating system of a wide variety, and Android system is one of the best. The number of applications based on the Android system has exceeded one million. The map application is essential software for mobile applications. At present, some of the map software provided by the store is more or less defects, such as the use of a wider Google map, because it cannot provide the offline data packet download, it will consume a large flow. In addition, a traditional map service general page or GPS navigator to work, these methods is not suitable for use in outdoor travel, and the cost is high, the operation is not convenient. Based on this, we developed a Android system under the multi-function map software, which is based on the high moral map provided free API for development, and can achieve three kinds of map view mode, but also to achieve offline map download and management, automatic positioning, hot search, bus route search, travel route search, advertising and weather forecasting and other functions.

\section{Construction of Development Environment}

The map software is an Ubuntu system, which is a desktop application based Linux operating system, which is built by a number of free software, and it can provide a new and fairly stable operating system for the general users. In the installation of Ubuntu 13.04, it is best to install the dual system, the first installation of Ubuntu and then install windows, this can be used to guide the Grub dual system, such as the system unfortunately lost, you can use Grub repair. PM partition magician on the hard disk partition, a Swap about the 3G partition and a 15G above the Linux system installed Ext3 or Ext4 partition. Eclipse programming tools and the Android plugin for eclipse ADT online installation and configuration real development environment, Android phone USB plugged into a computer to use command: sudo GEDIT /etc/udev/rules.d/ 51-android.rules, input the following content: SUBSYSTEM== "USB", ATTR $\{$ idVendor $\}=$ "mobile phone code", mode = "0666", which mobile code can through lsusb command to view. After the completion of the configuration, we should restart, and then the development environment has completed. 


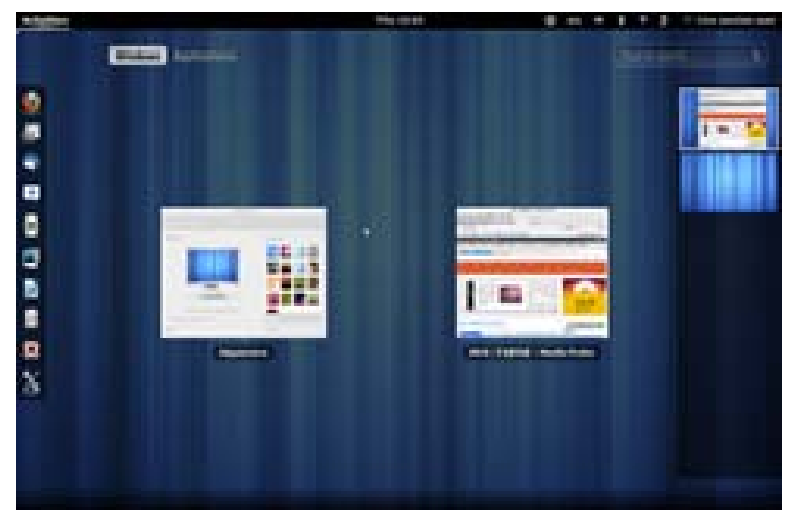

Figure 1 Ubuntu13.04 of Gnome on desktop

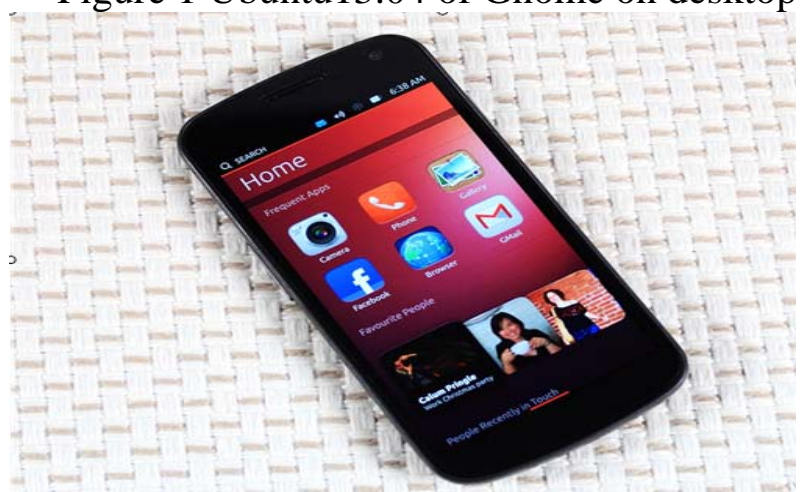

Figure 2 Ubuntu System of Smartphones

\section{Development of Map Software Based on Android}

\section{A: Function Modules of Software}

The map software is an independent application for Android mobile, including the number of functions. In the boot animation module, if it is the first time to run the software in addition to the animation, there are a few features to guide the page, for a brief introduction of the functions of the software. Menu can be left and right sliding, relying on hand gestures and touches to complete menu function, through the way of human-computer interaction to achieve the exit of the software, and make the interface more humane. Map module design 3 kinds of display modes: normal mode, real time traffic mode and satellite mode, and can set up automatic positioning and navigation in normal mode. In addition to viewing the city map, the software can also download and manage the offline city map, and its suspension, stop and delete functions, and can also be downloaded from the offline map display. In addition, in the software, a Mini browser is embedded in the software, and it can realize the function of the web page version of the map, and can integrate the web and Android technology to achieve a variety of web applications. Hot search features can search for the user's interest in things, such as KFC, hospitals, parks, gas stations, bus stops, etc. If it is a business partner, will also pop up the appropriate information. The weather forecast module provides a week of weather forecast ( 7 points per day), if there is a need to increase real-time weather. The module interface is provided by the weather and meteorological station of China in the JSON format, and the function of the weather forecast is realized by analyzing HTML. 


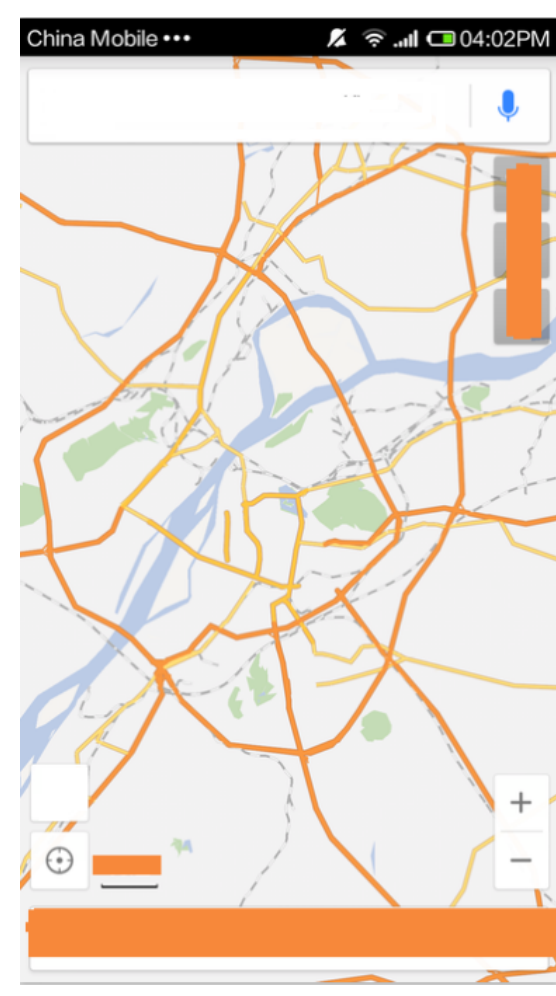

B: Function Design of Software

In the animation design, we disposed of modifying the Android source code or GIF parser to implement this kind of complex method. Here we use a simple comparison of a method, by taking the class as a tool, directly to the program, the change sheet resources, it can be very easy display GIF images; their realization of a class MyCustomView inherited View, the animation file with Moview.decodeStream parsed to create a Movie object in the constructor, get the current system time (SystemClock.uptimeMillis), and then get the length of time the current time setting animation, animation, redraw animation, then forced to redraw the current View to achieve animation the effect of the.

Sliding menu design can be said to be one of the difficult points in the design of the software. Through the sliding menu to achieve a relatively large number of interfaces, that is, the list of links. The design is through the GestrueDetector.OnGestureListener, OnItemClickListener, OnTouchListener, MyListViewFling, OnTouchListner and other interfaces to achieve. Click on a menu after a jump to the corresponding functions of the page, such as a click on the bus travel, transfer data through the Intent to the bus travel Activity. So you start another interface.

In the map display, when the map display module, the use of automatic positioning. MyLocation class by a method runOnFirstFi to send location information to the multi thread, and then by another thread to locate, so that the benefits of the software will not appear card dead phenomenon, as there is a need to locate the network, if the network is abnormal, if not used, it will lead to the program card. Android in a variety of ways to achieve the other thread and Main thread communication, using Android is the AsyncTask framework provides asynchronous processing auxiliary class, it can achieve time consuming operation in other threads, and processing results in the Main thread execution, for developers, it shielded the concept of multi thread and Handler. But the better the package, the higher the API, the more negative for the junior programmer, is that you do not understand its principle. When you need to face a more complex situation, and advanced API cannot be completed very HERSHEY'S, the problem came. So, we also need to grasp the more powerful, more free and Main thread communication method: Handler use.

Many functional modules in the input box is their own definition of MyEdit inheritance system Edit control, mainly is a copy of several methods. Here mainly to carbon OnDraw method, DrawBitMap expansion system edits only input words and not in front of the search icon button function. There are at least two ways to achieve this function, the first one is to insert a picture in 
the properties of the edit control, the second is the custom control of the software, the advantage is more flexible, it is also convenient for the future development of other software reuse.

The search results (such as merchant promotions information) on the map display in the software, the default settings for each page ten preferential information. In order to improve the user experience, so in the program to control the scope of the map, so that the results of the ten preferential information pages can be displayed in the current map view, if you click the next button, the next 10 points on the map displayed on the map. Search results are saved in getPage, the result method of the object can be returned to the last page of the data, and to keep it in the List list, for the process to do further processing. The result of each page is shown on the map by the addToMap method, and then we force the map and display it on the map. Click on a search result, a small window will pop up the corresponding information, such as address, phone number, etc.. This display is achieved by PoiOverlay.showPopupWindow () method, while the display window is a custom layout. The advantage of such a custom layout is that if you think this information window is not beautiful, you can modify the XML file and not modify the code itself, as opposed to the code, modify the configuration file to be easy.

In the travel route query module, the search process is set up by the Query object to set the starting point or end point, and the final result is obtained by the searchPOI method of the PoiSearch object. After the search will be saved in the PoiPagedResult object, and then draw the results to the map. Here to achieve the automatic scaling map, so that the results can be completely displayed on the screen. Due to the time consuming, it may also have other exceptions, so the results are not placed in the main thread, as far as possible to open up a new thread to draw. Here used the Message mechanism, through the message to notify the new thread drawing. Drawing is the result of drawing some more red dots, these points are custom cover MapPointOverlay.

The software provided by the weather forecast from the weather station in China to provide week weather Json data. The third party Java library jsoup is a HTML Java parser, through which the URL address and HTML text analysis. It provides a very convenient API, DOM, CSS and similar to the operation method of jQuery to remove and manipulate data, so as to realize the function of the weather forecast of the software.

\section{Conclusion}

This paper mainly designs a multifunction map software based on Android platform, which can realize three kinds of map mode, search and automatic location, hot search, bus route query, travel route search, advertising and promotion, weather forecast and so on. It has high application value. This paper expounds the design process and the realization of the software design of the software design. The development process has a certain reference to the design of the mobile intelligent map software.

\section{References}

[1] Lin Yulong, Xu Jianmin, Lin Peiqun, Chen Tiande, Map-matching Algorithm Research Based on Android Smartphone, J., Electronic Design Engineering, 10(2013)36-39+42.

[2] Wu Lin, Xiong Tao, Cai YongXiang, Design and Development of Map Application Based on Android Mobile, J., Urban Geotechnical Investigation \& Surveying, 2(2011)16-19.

[3] Wu Xianzhen, Design and Development of Application Software Module in Smart Phone Based On Android Platform, D., Harbin Institute of Technology, 2013.

[4] Xiong Gang, The Design and Realization of Smart Phone Based on Android, D., Wuhan University of Technology, 2010. 